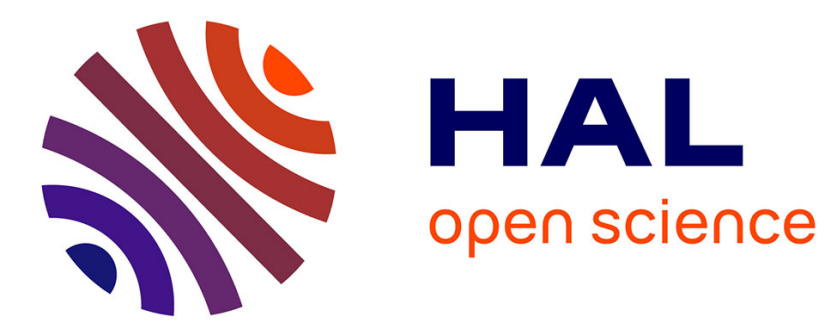

\title{
La sexualité, entre parole et parcours, des sujets infectés par le VIH
}

Ouriel Rosenblum

\section{To cite this version:}

Ouriel Rosenblum. La sexualité, entre parole et parcours, des sujets infectés par le VIH. Dialogue , 2011, Sexualité et parole, 3 (193), pp.115-124. 10.3917/dia.193.0115 . hal-01516932

\section{HAL Id: hal-01516932 \\ https://hal.science/hal-01516932}

Submitted on 24 Jun 2017

HAL is a multi-disciplinary open access archive for the deposit and dissemination of scientific research documents, whether they are published or not. The documents may come from teaching and research institutions in France or abroad, or from public or private research centers.
L'archive ouverte pluridisciplinaire HAL, est destinée au dépôt et à la diffusion de documents scientifiques de niveau recherche, publiés ou non, émanant des établissements d'enseignement et de recherche français ou étrangers, des laboratoires publics ou privés. 


\title{
La sexualité, entre parole et parcours, des sujets infectés par le viH
}

OURIEL ROSENBLUM

\begin{abstract}
RÉSUMÉ
Le sida, union de la mort et de la sexualité, rend celle-ci irreprésentable et a profondément modifié l'imaginaire sexuel. L'article s'efforce de montrer en quoi l'apparition du viH a redéfini la norme en cours au sein de la sexualité des individus, puis analyse les différentes étapes auxquelles sont confrontés les sujets séropositifs lors de leur parcours, avec le nécessaire réaménagement de leur sexualité ; il évoque ensuite l'aspect transgressif de la sexualité pour certaines populations et, enfin, donne la parole aux femmes séropositives sur le rapport à leur corps en relation à l'autre - corps sali qui souvent n'a plus droit au plaisir. L'infection au VIH vient rendre confus les différents registres tels que la mort, la vie, le sexe, elle engendre chez le sujet atteint honte et culpabilité : autant d'affects que le thérapeute doit accueillir pour redonner au sujet un plein sentiment d'exister.
\end{abstract}

\section{Mots-CLÉs}

Sexualité, vIH, transgression, normes, corps.

Aborder le lien entre sexualité et parole dans le cadre de l'accompagnement des sujets infectés par le VIH, en qualité de psychiatre psychanalyste au sein d'un service de maladies infectieuses, nous paraît paradigmatique de la difficulté d'exprimer le désir dans un contexte de contamination essentiellement sexuelle. Cette parole tue est bien le témoin de la culpabilité du sujet pris dans les rets de sa sexualité encombrée d'un virus, témoin d'une faute imaginaire, sous le sceau de la stigmatisation vécue comme irrémédiable. 
L'infection au VIH est une affaire de sang, de sexe et de mort et focalise des craintes réelles et imaginaires, constituant une surface de projection favorable à nos peurs. Le sida a à voir avec l'acte sexuel, avec les fantasmes qui présentifient le désir, avec la vie sociale. Le sida serait l'union de la mort et de la sexualité au niveau du réel et la sexualité qui peut devenir la mort n'est plus ainsi représentable.

L'apparition de cette infection a été productrice de savoir et de connaissance sur nos sociétés, en particulier sur la sexualité, avec la mise en évidence de la pauvreté relative de cette sexualité dans nos cultures, laissant ouverte la question : «En quoi le sida a-t-il modifié l'imaginaire sexuel ? » Mais, assez rapidement, le sida s'est constitué comme champ en se banalisant et se remédicalisant, en induisant l'identification de populations distinctes comme celles des mères, des enfants, des toxicomanes, des homosexuels, des migrants d'Afrique... Cet aspect de l'accès aux soins s'ouvrait plus largement à des phénomènes de société tels que la requalification d'un acte sexuel ou bien la reconnaissance légale du couple homosexuel, jusqu'au débat concernant le statut de l'homoparentalité, redéfinissant la fonction parentale et la place de l'enfant, engageant la place des filiations instituées et biologiques.

Dans cet article nous verrons en quoi l'apparition du VIH a redéfini la norme en cours au sein de la sexualité des individus, ensuite nous analyserons les différentes étapes auxquelles sont confrontés les sujets séropositifs lors de leur parcours, avec le nécessaire réaménagement de leur sexualité, puis nous évoquerons l'aspect transgressif de la sexualité pour certaines populations ; enfin, nous donnerons la parole aux femmes séropositives dans leur rapport à leur corps en relation à l'autre.

\section{VIH, normes et sexualités}

Depuis les années 1980, le sida a modifié les représentations sociales et les normes de la sexualité. Nous avons assisté à la mise en place d'une nouvelle échelle de valeurs issue, cette fois, de l'épidémiologie, en contraste avec l'échelle de valeurs morale, idéologique ou culturelle, qui entoure la sexualité. Ainsi, selon A. Spira et N. Bajos qui ont mené une étude sur les comportements sexuels en France, la pratique sexuelle considérée comme normale, à savoir la pénétration génitale, est mise sur le même plan, au niveau des pratiques à risque, que la pénétration anale. D'autre part, les perversions sexuelles, en dehors de la sodomie, sont à moindre risque que les pratiques dites «normales », voire sans risque pour certaines d'entre elles.

En revanche, pour l'écoute du psychanalyste, le risque majeur dans une relation sexuelle, ce n'est pas le sida, mais l'autre et la crainte de le perdre. Nous nous sommes posé les questions, ici, de notre fonction vis-à-vis des pratiques sexuelles de nos patients. Quelle place occupons-nous, dans ces actions de 
prévention qui reposent sur le postulat que les individus souhaitent vivre et se protéger? Que fait-on de sa subjectivité, de son désir que l'autre se protège ? Que penser de notre neutralité face aux individus qui font courir un risque à leur partenaire ? Quelles modifications du rapport sexuel implique le port du préservatif, avec le renforcement du phallocentrisme et la question de la sexualité en institution ? Tant que le VIH demeure une maladie du secret, de la sexualité, et de celle-ci perçue comme différente, les patients fascinent encore la société.

\section{Sexualité et parcours du sujet infecté}

L'expérience de Roland Tubiana, médecin infectiologue avec qui nous collaborons, a été très précieuse pour appréhender le cheminement des patients séropositifs confrontés aux différentes étapes de leur infection. Pour ce médecin, la sexualité est présente, discutée et en constante évolution à tous les moments de la vie des sujets infectés par le $\mathrm{VIH}$ : avant l'infection, par les mesures de prévention recommandées, essentiellement par le port du préservatif ; au moment traumatique de l'annonce du diagnostic de la séropositivité, par la remise en cause de l'individu dans son intégrité physique et psychique et le bouleversement de ses relations intimes avec ses proches et son environnement.

Un sujet infecté nous a dit : «Ce qui est fondamental, c'est le regard qu'on porte sur la personne. Est-ce qu'il y a de l'humanité ? La mise à l'écart, c'est tout cela aussi qui se ressent. Et je crois qu'au moment de l'annonce de la séropositivité on ne comprend pas grand-chose de toute façon. La seule chose dont on a besoin, c'est déjà d'être accueilli dans cette annonce problématique. Et pouvoir y revenir afin que l'on nous l'explique dans un second temps, parce que vouloir tout nous expliquer à ce moment-là, cela ne sert à rien, on est en état de choc. »

Lors de l'évolution de l'infection, les sujets mettent en avant les stigmates et les doutes induits par les résistances au traitement qui modifient l'enveloppe corporelle, avec leur cortège d'inquiétante étrangeté. En revanche, sous traitement antirétroviral, par les effets d'augmentation de l'espérance de vie sans maladie apparente, les sujets arguent de la qualité de vie retrouvée, au point d'exprimer des désirs de parentalité et ainsi de vouloir vivre désormais comme les gens «normaux » à leurs yeux.

En interrogeant les médecins infectiologues, on notera chez eux la difficulté à faire émerger les besoins et les attentes, en termes de sexualité, des personnes infectées par le vIH. Par ailleurs, les conseils de prévention, comme celui d'éviter les rapports pendant les règles ou bien la sodomie, sont souvent considérés comme une stigmatisation supplémentaire, une exclusion et un énième rappel de leur infection. La question de fond concernant la satisfac- 
tion de la vie sexuelle est souvent éludée par des considérations autour de la prévention et des conseils médicaux donnés par des intervenants démunis ou non préparés aux questions des patients.

Lorsque le sida est apparu, il y a vingt ans, être infecté était associé à une sexualité marginale, atypique et parfois honteuse. Cette sexualité était vécue comme un vecteur de maladie et de mort, ce qui a pu entraîner chez certains des comportements paradoxaux extrêmes, faisant courir des risques chez leurs partenaires, la pulsion de mort, ici décuplée, faisant son œuvre.

Actuellement, les traitements adaptés permettent un contrôle efficace du virus, une restauration quasi parfaite des réponses immunitaires des patients et par conséquent une espérance de vie sans pathologie à long terme. Cette chronicité sous condition, si elle redonne espoir, fragilise certains individus qui sont rappelés à l'ordre par le virus de façon radicale lors de chaque nouvelle relation sexuelle. Depuis l'instauration de traitements efficaces, les sujets infectés peuvent normaliser les champs de leur vie sociale ; mais qu'en est-il de leur sexualité ?

Souvent, après l'annonce traumatogène de la séropositivité, une période de désinvestissement libidinal s'installe durablement, s'accompagnant d'une sidération des processus psychiques et d'une abstinence prolongée. Ici, les questions formulées concernent le mode de contamination, son existence en compagnie du virus, la vie de couple quand l'un est infecté et l'autre pas, le secret vis-à-vis de l'entourage. Le sujet devient un acteur qui aurait le mauvais rôle dans le trio des composantes de la transmission : une personne porteuse du virus, un rapport sexuel et l'absence de protection. La sexualité, même non déclarée ni assumée, est omniprésente : cause fréquente de l'infection, affectée par la pathologie et les traitements, mais également marqueur indélébile de la différence.

Les problèmes de sexualité jalonnent donc le parcours de vie de la personne infectée par le vir. Les troubles de la sexualité sont fréquents (25 à $70 \%$ selon les études), tant chez les hommes que chez les femmes et quelle que soit l'orientation. Les hommes se plaignent davantage de baisse de la libido et de la performance, d'impuissance, s'interrogent sur les effets secondaires des traitements antirétroviraux et réclament des médications contre les troubles de l'érection. Les femmes expriment davantage les difficultés soit à aborder le sujet avec les partenaires avec qui elles partagent leur vie affective et sexuelle, soit à investir libidinalement leur corps sexué.

Les individus rencontrés peuvent affirmer leur normalité, malgré la présence du virus, via la reprise d'une activité sexuelle : «Je ne dis rien, sauf si le (la) partenaire le demande ; je ne vais pas sauver le monde » ou, à l'opposé, exprimer une culpabilité ou un sentiment de honte: "Je ne veux aucune vie sexuelle, c'est trop difficile d'en parler et je ne veux pas être responsable de l'infection d'une autre personne, je ne veux pas faire à une autre personne ce 
que l'on m'a fait. » Ce dernier sentiment, qui conduit à l'abstinence sexuelle, est souvent lié à une souffrance psychique profonde et à une dégradation de l'estime de soi. Heureusement, ce choix peut être remis en cause dès lors que le temps est investi dans sa signification de porteur de vie sans maladie, sous traitement, ou bien à l'occasion d'une rencontre amoureuse.

La recension des plaintes exprimées traduit un vécu fantasmatique de discrimination sexuelle : "Je ne veux pas que l'on me regarde comme un pestiféré », "Je ne veux pas vivre la fuite de l'autre », "Si je le dis, il s'en va et si je ne le dis pas, je me sens coupable et lâche », «On dit que le traitement permet une vie normale, ce n'est pas vrai au niveau de ma sexualité », "Comment voulez-vous que je trouve un(e) partenaire pour faire ma vie ?»... Le statut sérologique exempt du virus, être séronégatif, inaccessible dans les faits, est vécu comme un sésame de la vie sexuelle épanouie. Ces appels peuvent amener l'infectiologue à proposer une prise en charge par les psychologues, psychiatres ou encore sexologues.

\section{Sexualité et transgression}

Dans certains milieux homosexuels à partenaires multiples, la prise de risque des partenaires séronégatifs est parfois consciente, voire revendiquée, associée aux comportements de type initiatique ou ordalique comme celui de s'assurer la valeur de l'existence et d'éloigner l'angoisse de mort. Ici, la transgression et le risque sont le moteur du plaisir et de la jouissance dans l'acte sexuel non protégé. Mais on peut retrouver, dans cette compulsion à agir, un sentiment de solitude, un mal-être avec une consommation d'alcool et de psychotropes à visée désinhibitrice et anxiolytique. On peut relever également des épisodes de fragilité au sein de la vie de couple, l'abandon de la protection quand la relation devient stable, l'établissement de relations en dehors du couple.

Pour les populations migrantes africaines, la contamination hétérosexuelle est la plus courante. La protection du ou de la partenaire fait l'objet de discussions longues et délicates avec les soignants, dans la mesure où l'acte de procréer est primordial, surtout chez la femme. Par ailleurs les femmes africaines séropositives n'osent pas bénéficier du soutien des associations par crainte d'être stigmatisées, le VIH est encore lié ici à une sexualité déviante. Chez un couple africain, quand il y a une annonce de contamination, on accuse la femme d'avoir ramené le virus à la maison ; elle est alors considérée comme une prostituée. Ainsi, quand les femmes africaines apprennent leur séropositivité, elles la cachent de peur d'être rejetées, ce qui équivaut, pour elles, à une mort sociale. 


\section{Sexualité et paroles de femmes séropositives}

Nous allons rapporter quelques paroles de femmes séropositives entendues lors d'un atelier au cours d'un forum organisé par plusieurs associations (Act Up Paris, Aides, le Kiosque info sida toxicomanie, le Mouvement français pour le planning familial, Sida Info Service), qui a été mis en place afin que cesse une certaine invisibilité des femmes confrontées au VIH.

Ainsi, cette femme séropositive depuis vingt ans nous a parlé de ce qui était le plus difficile dans son parcours : l'annonce de la séropositivité - comment le dire à son partenaire ? Certaines ont vécu le rejet, d'autres le redoutent et d'autres encore ont été abandonnées par leur compagnon dès les premiers signes d'aggravation de la maladie. La culpabilité est forte de blesser le partenaire et de l'angoisser, tout comme la crainte de ses réactions quand il apprendra la nouvelle. Dès l'annonce de la séropositivité, la crainte de la contamination est omniprésente et elle le restera. Elle engendre la peur de certaines pratiques et vient modifier en profondeur l'organisation et l'intimité du couple. Il faut désormais protéger l'être aimé. C'est une étape nouvelle qui interroge la confiance en soi, le rapport de son corps à soi, à l'autre et au plaisir. Si le partenaire n'est pas contaminé, il faut gérer le trouble quand la question du préservatif se pose - sans parler de la peur d'une rupture de préservatif.

Choisir librement un partenaire n'est plus vraiment possible, car la sensation de ne plus avoir droit à l'amour s'impose. C'est difficile de se dire qu'il faut se protéger à vie. Un médecin sexologue souligne à quel point les femmes séropositives sont seules et souffrent en silence. Elles sont dans l'impossibilité à dire, à vivre, y compris leur sexualité, la peur de rejet domine. Une femme a dit que si un homme l'a contaminée, un autre lui a redonné le goût de vivre, insistant sur son besoin d'amour pour vivre et prétendant que la solitude est pire que la maladie elle-même. Ainsi, l'élaboration collective, à partir de groupes de parole, permet de s'extraire d'un fort sentiment d'isolement où l'on finit par penser que l'on est seul à ressentir ce que l'on vit.

$\mathrm{Au}$ sein du couple, lorsque les femmes sont séropositives, elles assument leur maladie ; mais si elles estiment avoir droit à une sexualité, quelque chose est cependant mort en elle. Elles disent avoir de moins en moins de désir et souffrent de dyspareunies d'origine psychogène qui se traduisent par des douleurs lors de la pénétration. Souvent, elles ne reconnaissent plus le caractère érogène de leur corps sexué qui a subi des transformations du fait de la maladie et des effets des traitements antirétroviraux. Les jeux de l'amour et de la séduction ne sont plus au rendez-vous. Certaines femmes abordent des problèmes de sécheresse vaginale dus à des dérèglements hormonaux propres à l'infection ou aux traitements ; cette situation accroît leur crainte et les risques de rupture du préservatif. 
Même après des années de traitement, le plus difficile à vivre pour elles, c'est le regard porté sur soi, son propre corps, et celui de l'autre sur soi à affronter. Le corps infecté est souvent synonyme de souillure, l'objet de sentiments obsessionnels autour de la saleté et de rituels compulsifs jusqu'à une certitude, de nature hypocondriaque mélancoliforme, d'être pourrie de l'intérieur. Ce moment de partage avec l'autre est très important, à la fois recherché et redouté. Une femme nous a dit : «À l'annonce de ma séropositivité, je me sentais sale. Je pouvais prendre ma douche dix fois par jour, je me lavais, encore et encore, surtout mon sexe, et puis je me sentais comme... je ne sais pas. Je demandais sans cesse à la personne qui était près de moi : "Est-ce que tu sens que j'ai une mauvaise odeur ?" ; il me disait que non, mais moi je lui répondais que je sentais mauvais, ce qui me contraignait à me laver à longueur de journée. »

L'apparition des traitements peut être déterminante dans le changement du rapport à soi et aux autres. Nous rapportons les propos d'une femme séropositive : "Pour moi, le fait que la charge virale devienne indétectable dans mon sang a joué un grand rôle. Je me sentais beaucoup moins toxique, moins contaminante, j'étais quand même moins flippée. Et je pense que ce sentiment de souillure dépend également de l'époque où l'on a appris sa contamination. Pour ma part, j'ai été contaminée dans les premières années où tout a été dit sur cette maladie, tout et n'importe quoi, et nous avons été stigmatisés de façon atroce. À cette époque, nous étions vraiment des moins que rien, des pédés, des toxicos, des salopes. Je pense qu'aujourd'hui ce n'est pas plus facile d'être séropo, mais il y a quand même des images qu'on ne véhicule plus de la même façon. »

Pour d'autres, le préservatif, c'est un obstacle physique entre soi et l'autre, une ambivalence difficile à vivre entre l'impossibilité et l'envie de s'abandonner à la force du désir. La crainte d'oublier le préservatif dans un moment de désir intense est très présente. La peur qu'il craque, dont on a déjà parlé, est permanente, elle engendre la lassitude, la sensation de ne plus être une femme, mais seulement un objet. Le préservatif finit par devenir un obstacle au ressenti de son propre corps et au corps de l'autre, remettant en cause le droit d'aimer et d'être aimé. Cette protection obligatoire interfère dans le jeu du désir et l'interrompt brutalement. Son retrait est obligatoirement rapide, il engendre une frustration permanente avec laquelle il faut apprendre à vivre.

Toutes ces craintes peuvent converger vers l'arrêt de la relation sexuelle ou vers la rencontre d'un partenaire séroconcordant, avec l'illusion que le partage de la séropositivité puisse offrir un espace où l'intime se confond avec le même statut biologique : on est dans le déni de la différence des sexes, avec son cortège de malentendus. Quelquefois, la peur du sentiment amoureux est très présente. Les femmes insistent sur la nécessité de se dégager de la parole des experts en prévention, qui leur rend la sexualité impossible à vivre au quotidien. Elles expliquent que, tant qu'elles sont dans « ne faites pas ci, ne 
faites pas ça », rien ne prend de sens pour elles. Il faut, disent-elles, inventer ensemble une nouvelle vie. Elles déclarent souhaiter développer un esprit critique vis-à-vis de l'information délivrée concernant la prévention des risques de contamination. Elles disent être soumises à des formulations contradictoires et appellent de leurs vœux l'aide des associations accueillant la parole des sujets séropositifs. En effet, face à tous ces discours, afin de brasser toutes les informations, elles éprouvent la nécessité d'investir des lieux collectifs, espaces de débat, controverse ou consensus. Ce droit à l'information doit évidemment être accompagné par une pédagogie qui viserait à exercer un esprit critique pour amorcer une nécessaire distanciation. Cela participe à un travail de recomposition quand on a perdu tous ses repères.

\section{Ouvertures}

La présence du VIH témoigne de la nature non domestiquée, dangereuse et source de désordre, évocatrice du désir sexuel incontrôlable. La pandémie du virus peut s'apparenter à une sexualité généralisée qui s'adresse aussi bien à l'hétérosexualité la plus caricaturale (la polygamie, le vagabondage sexuel, la liberté sexuelle...) qu'à une sexualité déployée dans une perspective transversale du développement libidinal. En effet, le viH décline les avatars de la sexualité polymorphe (anale, homosexuelle, bisexuelle, hétérosexuelle, perverse...) ou ceux de leur étayage sur les besoins (toxicomanie). Le désir sexuel est perçu comme une menace potentielle contre les unions légitimes et contre les normes de la société, ses coutumes, sa loi. Le social ne peut s'établir que sur la base du sacrifice de quelque chose qui est profondément contenu dans la sexualité humaine - et qui est son caractère fondamentalement asocial, nous dit M. Godelier. L'humanité est contrainte de fabriquer du social avec du sexuel qui se prête à toutes les métamorphoses que lui font subir les cultures. Avec le sexe, on peut jouir de soi, des autres, faire jouir autrui. Mais le sexe est le siège également de la frustration, de la souffrance et de la violence. Ainsi la sexualité est le lieu privilégié du corps où se soudent la logique des individus et celle de la société, où s'incorporent des symboles, des désirs et des intérêts opposés, organisant le refoulement-travestissement de tout ce qui, dans la sexualité, n'est pas compatible avec les activités conscientes des individus en société.

Le virus, témoin primitif de la sexualité antérieure, peut être l'incarnation d'un biologisme spontané, fruit du rapport au vivant, à l'animal, avec l'idée que l'animal est au plus profond de l'homme. Selon J. Laplanche, ce renvoi à l'animal est purement idéologique, il nous permet de nous décharger de notre inconscient en l'attribuant au non-humain en nous. Pour cet auteur, les théories biologisantes sur notre sexualité demeurent des codes de traduction mis à la disposition de l'enfant par l'univers culturel pour lui permettre de tenter de 
traduire les messages adultes. Les théories référant l'homme au vivant visent à maîtriser le sexuel pulsionnel qui infiltre la relation adulte-enfant.

Mais la sexualité humaine n'ayant plus rien à voir avec le sexuel reproductif proposé par la nature (elle est par définition « dénaturée »), celle-ci a contraint l'homme à devenir coresponsable avec elle-même de sa propre évolution. Le VIH, témoin de cette sexualité dénaturée, peut-il contribuer à coresponsabiliser la nature associée à l'homme ? L'autodomestication par l'homme de ses pulsions, à l'origine, selon Freud, de l'éternel malaise dans la culture, ne semble jamais rencontrer d'issue. Mais peut-elle obtenir un exutoire à travers le processus de sublimation ?

C'est au moment où ils ne sont plus victimes d'une tragédie que se posent pour ces sujets les questions de la vie. Il s'agit du temps de la normalisation, où l'accès à la relation sexuelle pour ces patients est venu signifier que le virus, porteur de désintrication pulsionnelle et de finitude pour l'individu infecté, a été domestiqué. Mais, chez certains sujets, il peut exister un lien entre fragilité narcissique et sentiment d'exister. Notre travail est d'accueillir les affects et de favoriser la reconnaissance par le sujet de l'ambivalence de ses sentiments : ainsi pourra-t-il ressentir l'affect et le nommer. Par ailleurs, l'infection au $\mathrm{VIH}$ vient rendre confus les différents registres tels que la mort, la vie, le sexe. Il nous faut donc ouvrir un espace de la différence pour relancer le processus de subjectivation, dans une approche clinique connectée avec l'histoire de la maladie. Toute affirmation de vie de la part de ces sujets, qui la vivent comme menacée, est vécue comme porteuse d'agressivité, alors qu'un projet de couple apparaît comme une tentative d'anticipation qui les délie des sentiments de honte et de culpabilité. La honte serait le premier temps de la culpabilité, vis-à-vis du regard de l'autre sur soi, alors que l'objet d'amour serait l'objet de soi-même entraînant un réveil narcissique. Ainsi l'entourage qui incarne l'histoire clinique du sujet va-t-il être un des regards de l'autre par lequel le sujet va se construire en partant de la question : suis-je malade ou pas et, si je ne le suis pas, qui suis-je ?

Ouriel Rosenblum

Psychiatre psychanalyste

Services des maladies infectieuses, maternité

et psychiatrie de l'enfant

Pitié-Salpêtrière, Paris13 ${ }^{\mathrm{e}}$

Professeur de psychologie clinique et psychopathologie,

Université de Bourgogne rosenblouriel@noos.fr 


\title{
Bibliographie
}

Godelier, M. 2001. «La sexualité est toujours autre chose qu'elle-même », Esprit, 273, Paris, p. 96-104.

LaPlanche, J. 1999. La sexualité humaine, biologisme et biologie, Paris, Les Empêcheurs de penser en rond.

Pommier, F. 1996. La psychanalyse à l'épreuve du sida, Paris, Aubier.

Rosenblum, O. 2007. «Sexualité risquée, procréation assistée », Études freudiennes, horssérie, D. Brun (sous la direction de), «La place de la vie sexuelle dans la médecine », Paris, p. 299-306.

SprRA, A. 1993. (sous la direction de). Les comportements sexuels en France, Paris, La Documentation française.

Tubiana, R. 2007. « La sexualité dans le parcours des patients infectés par le viH », Études freudiennes, hors-série, D. Brun (sous la direction de), « La place de la vie sexuelle dans la médecine », Paris, p. 153-158.

États généraux 7 mars 2004, Femmes et Sida, Inter associatif, Paris.

\section{SEXUALITY, BETWEEN SPEECH AND STAGES IN LIFE, OF HIV-INFECTED SUBJECTS}

\begin{abstract}
AIDS, that union between death and sexuality, makes the latter non-representable and has deeply modified the world of sexual imagination. The article seeks to show how the emergence of HIV has re-defined the current norm within individuals' sexuality and then goes on to analyse the different stages HIV positive subjects have to confront as they go through life, with the need to re-organise their sexuality; it then evokes the transgressive aspect of sexuality for some sectors of the population and, finally, gives HIV positive women the opportunity to speak out on how they come to grips with their bodies in relation to partners - soiled bodies henceforth disentitled, deprived of pleasure. HIV infection has the effect of sowing confusion between the different registers such as death, life and sex, and causes in the subject feelings of shame and guilt: so many effects that the therapist has to take on board to render the feeling that they exist in full to the subject.
\end{abstract}

\section{KeYWORDS}

Sexuality, HIV, transgression, norms, body. 